\title{
RADIATION PROTECTION IN PEDIATRIC RADIOGRAPHY - INTRODUCING SOME IMMOBILIZATION AND PROTECTION EQUIPMENT
}

\author{
Dejan KLAVS*, Dženana PAŠAGIĆ, Nuša KOTAR
}

University Clinical Centre, Children's Hospital Ljubljana, Radiology Unit Ljubljana, Slovenia

\footnotetext{
*Corresponding author: dejan.klavs@gmail.com Tel.: + 38615229264

Fax.: + 38613077785
}

Received: November 1, 2015

Accepted: December 24, 2015

Key words: Children - Immobilization equipment - Radiation protection equipment - Safety.

\begin{abstract}
In this article immobilization equipment and safety measures used during radiographic imaging in childhood are presented. Pediatric radiologists and radiographers should work together to provide a child friendly environment during imaging, to minimize the stress for the patient and parents, and to facilitate the child's cooperation. The maximum information possible must be gained from the radiographic procedure. Considerable skill and experience are required to obtain the best possible image in the shortest possible time. It is the responsibility of the radiology department to use proper imaging protocols and parameters for the size of the child. Equipment in a child friendly imaging department needs to be user friendly. The visual appearance of the equipment is also important to make it less frightening for children. It is important to choose the proper ancillary equipment for immobilization during radiographic procedure and explain to parents the importance of using it. The main goal of the immobilization equipment is to prevent unwanted movement, thereby shortening the time of the procedure, avoiding unnecessary repetitions of radiographic procedures and thus enlargement of the dose. At the same time, immobilization and protection equipment helps to avoid unnecessary exposure of parents taking part in radiographic procedure to ionizing radiation. Conclusion Proper use of children's immobilization and radiation protection equipment, together with an adjusted imaging technique, results in a quicker radiation procedure and lower radiation dose.
\end{abstract}

\section{Introduction}

Even though radiation is part of our environment, it can be used for good purposes in medical services. When using radiation in medical diagnostics the risk on the one hand and the benefit on the other are considered (1). The principles of radiation protection in medicine apply wherever ionizing radiation is used for diagnosis or therapy. This is particularly important in the case of pediatric patients, because children are more sensitive to radiation exposure than adults, and have a longer potential lifespan in which radiationinduced diseases may appear decades later. Risks associated with ionizing radiation can be divided into the stochastic and deterministic effects (2). Deterministic effects have a threshold dose below which the effects do not occur (>0.1 gray-Gy): the threshold dose for skin reddening is 3 to $6 \mathrm{~Gy}$, for detectable 
opacity of the eye lens 0.5 to 2 Gy, for temporary hair loss $4 \mathrm{~Gy}$, and for permanent sterility 2.5 to 6 Gy (3). They generally occur after high-dose exposures and are most important in radiotherapy and high-dose interventional procedures, but they are not expected in diagnostic procedures (2). Stochastic effects are also considered (4). The probability of their occurrence, but not their severity, depends on the radiation dose. Stochastic effects are more random, which means that the occurrence of individual events cannot be predicted, and it cannot be determined whether genetic changes or induction of cancer may occur, regardless of the dose absorbed. There are no threshold doses above which these effects will definitely occur (5). Stochastic radiation risks are carcinogenesis and genetic effects. Children are ten times more sensitive to the stochastic effects of ionizing radiation than adults, due to the higher rate of dividing cells (6). This is the reason why special attention should be paid to children when using radiation.
In this study immobilization and radiation protection equipment are presented, including their purpose (prevention of unwanted movement) and role (optimization of radiation exposure) during different radiographic procedures.

\section{Immobilization equipment}

Both adults and children usually fear the unknown. For a child, diagnostic imaging represents a world of equipment, strangers and shiny technology, and most departments are noisy and busy. First of all, the environment should be made as friendly as possible for children, and a sense of trust should be developed between the radiographer, the child and the parents. Parents are actively involved in the procedure: they calm, comfort, and undress the baby, if necessary. Before using immobilization ancillary equipment, it is necessary to take some time and explain to the parents why (to prevent unwanted movement and repetition of procedure) and how

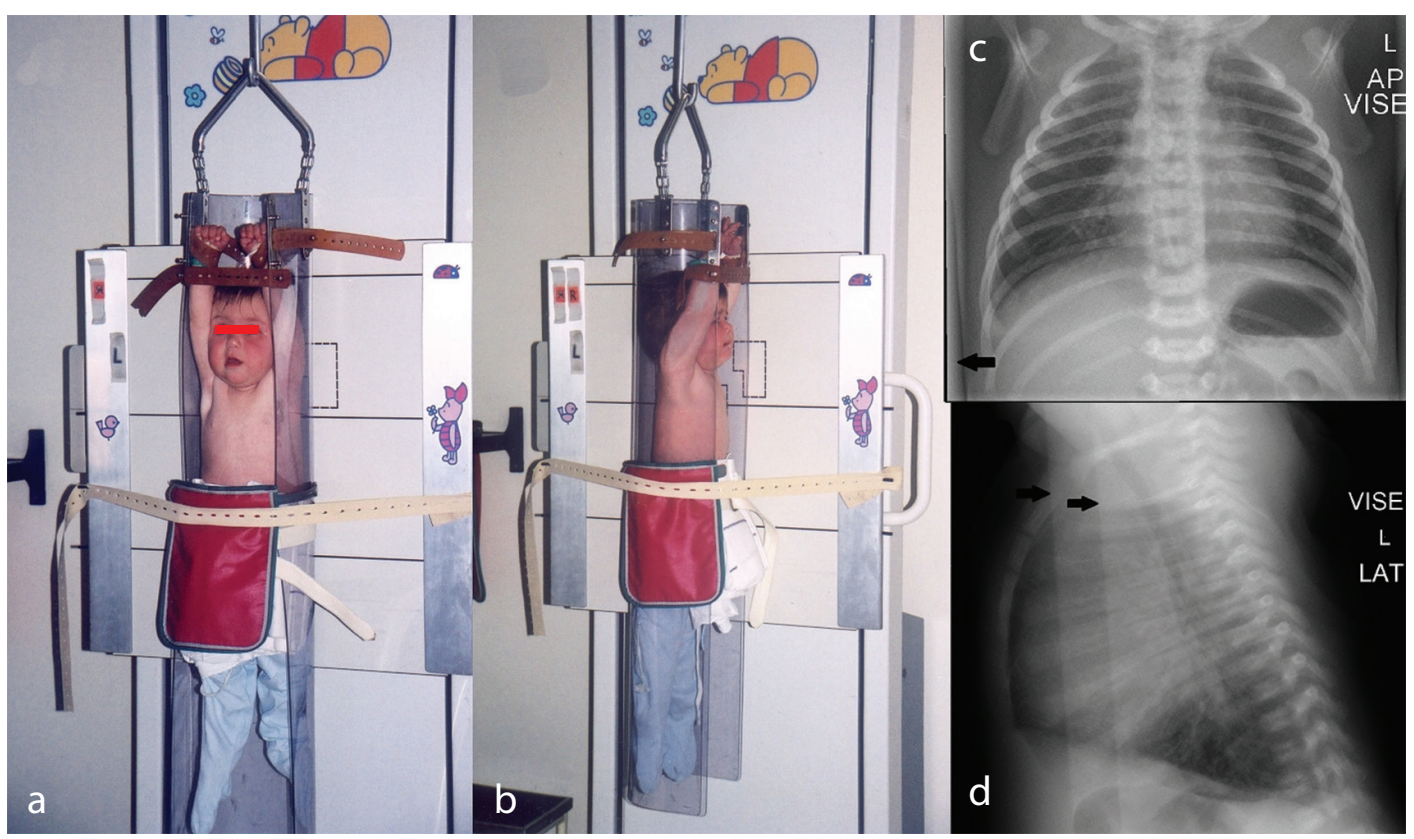

Fig. 1 Chest X-ray immobilization ("baby-fix"): position and fixation of the baby (raised arm, lead rubber apron for gonadal protection) in a) antero-posterior and b) lateral position. The shadow of equipment seen on c) antero-posterior and d) lateral radiograms (black arrows). 


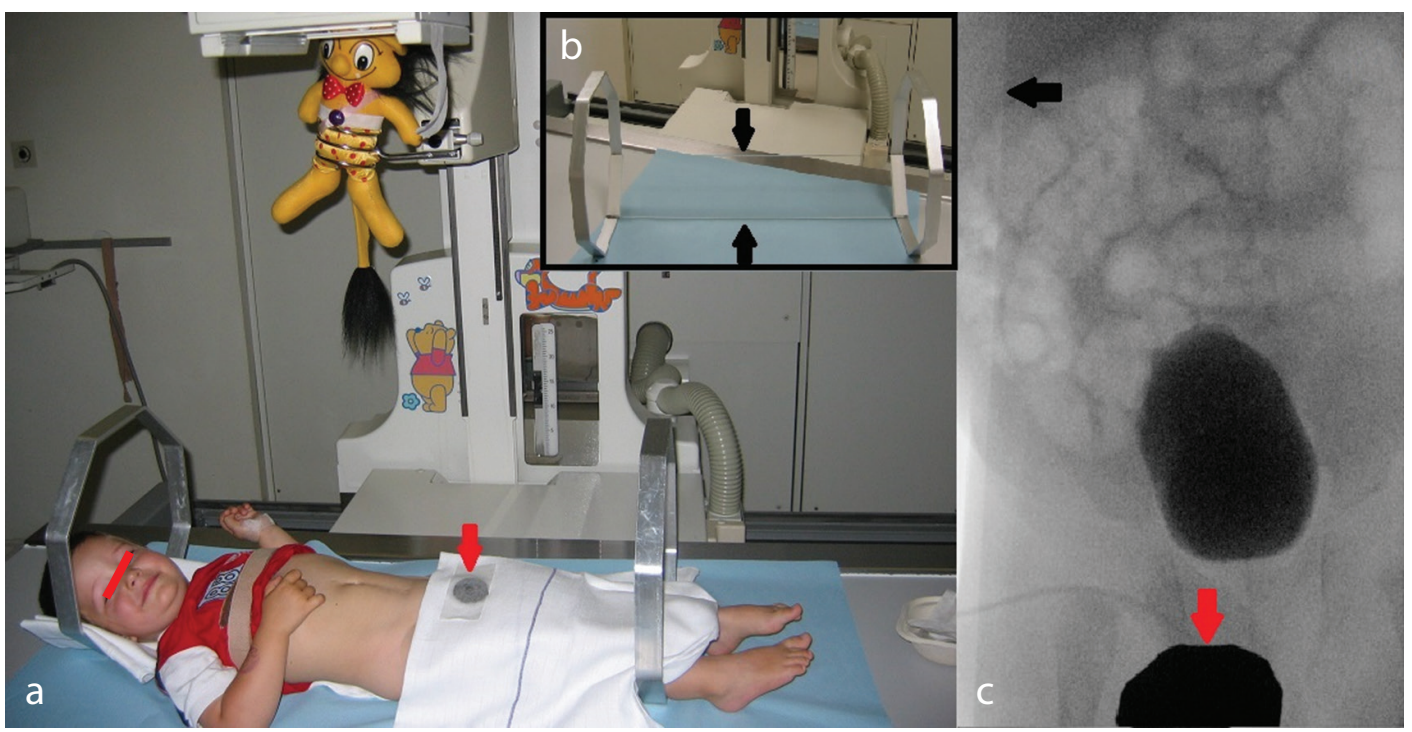

Fig. 2 Ancillary immobilization equipment for voiding cystourethrography: a) and b) Plexiglass fixator (black arrows), gonad shielding made of lead rubber (red arrow), c) shadows of immobilization equipment seen on radiogram.

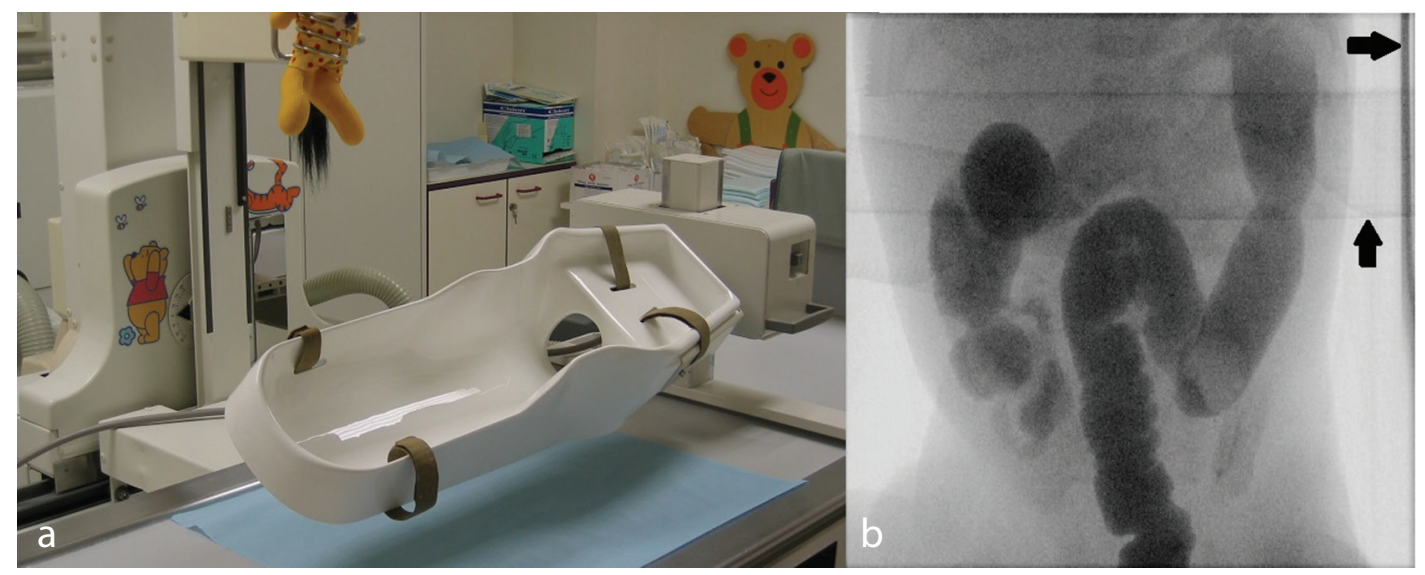

Fig. 3 Mulda: a) immobilization equipment used for contrast imaging of the gastrointestinal tract (specially designed shape). Working environment adapted for children with toys and stickers, b) shadows of immobilization equipment seen on radiogram (horizontal arrow and fasting strap - vertical arrow).

particular ancillary equipment is used during the radiographic procedure. Immobilization is performed by the radiographer. During the radiographic procedure, parents are asked to leave the examination room. Sometimes, if an ancillary tool cannot be used, one of the parents (usually the male, avoiding any unknown pregnancy in the woman) is asked to hold his/her child in the requested position. In this case, the parent has to use personal protection equipment (lead coat, thyroid collar and lead glasses) (1).

The main purposes of good immobilization, proper use of radiation protection and adjusted imaging techniques are to shorten the duration of the radiation procedure, avoiding repetition, and thus limiting the children's exposure to radiation. Immobilization equipment can be seen on the radiogram as a darker shadowed area, but is does not 
affect the diagnostic evaluation of the radiogram. The role of the radiographer is to use the appropriate positioning of the child, when using immobilization tools, without affecting the diagnostic purpose of the imagining. (Fig. 1c and 1d; Fig. 2c; Fig. 3b). The quality of immobilization instruments has to be regularly checked to ensure their proper functioning, so that they are safe for use. The most important immobilization equipment used in childhood is described below.

\section{Chest $x$-ray immobilization}

Chest x-ray immobilization, known as "baby-fix", is an ancillary tool, mostly used for chest $\mathrm{x}$-ray imaging in pediatric radiology. It may also be used for head and spinal imaging (1). It is made of Plexiglass and is shaped like a tube with rubber straps, making it possible to fix the child safely. Depending on the size and the weight of the child, different sizes of chest x-ray immobilization are available. Correct positioning of the child in the chest $\mathrm{x}$-ray immobilization equipment is important to obtain useful diagnostic chest radiography. This is achieved by placing the child in a "baby-fix" with the hands raised over the head and binding them with rubber straps around the wrists. By doing this the shoulder blades are projected outside the lung area. Then the body of the baby is stretched and aligned with the legs. Symmetry of the chest should be kept in mind. A foam pad is placed on the lower part of the abdomen and fixed with straps. Finally the head is fixed above the forehead. The "baby-fix" with the child inside is hung in front of the detector and fixed with a rubber strap. The abdomen and gonads are shielded with a lead rubber apron (Fig. 1). All these provide the optimal conditions for imaging. Due to the influence of the $0.5 \mathrm{~cm}$ perspex, the radiographer should slightly increase exposure factors. The radiographer may use automatic or manual exposure. When using automatic exposure, a middle ionization cell must be selected, due to the small investigation area. Also, accurate centering is of great significance, so that exposure may be interrupted on time. Close collimation on four sides onto the outer chest margins and shielding the gonads must be kept in mind.

\section{Immobilization equipment for voiding cystourethrography}

The child is placed in a specially designed Plexiglass fixator and fastened with stripes over thorax and thighs (Fig. 2). It allows a quick turn to the lateral position during urethra evaluation. It is possible to achieve correct positioning and collimating the field of vision only to the area of interest. For boys the gonads shields of different sizes are used, while for girls protection of the gonads cannot be used, because it could cover part of the examination (1). Proper fluoroscopy techniques are required to minimize the baby's exposure to radiation. Nowadays pulsed fluoroscopy is used, because a lower radiation dose can be achieved compared to continuing fluoroscopy. Pulses at the lowest possible rate (less than 5 pulses per second) and a narrower pulse width, reduce radiation exposure. During the procedure, the radiographer can change the pulse rate depending on the needs of the procedure. For example, when evaluating voiding, a higher pulse rate is required $(3,7)$.

\section{The immobilization equipment for gastroenterological imaging (mulda)}

The immobilization equipment using during contrast imaging of gastrointestinal tract is made of Plexiglass and has a specially designed shape (Fig. 3). It is called a "mulda". The contrast medium may be administered per os or through a rectal tube (using the hole 
on the bottom of the mulda) by a trained nurse. The mulda is connected to a handle which allows movement in various directions (up, down, $360^{\circ}$ rotation), so the radiologist is able to monitor the contrast medium in different projections. Two sizes of mulda are available on the market, depending on body mass. The smaller one is used for babies up to 6 kilograms and the larger one for babies up to 15 kilograms (1).

The child is placed in the mulda, fastened with straps over the ankles and both hands, and, if necessary, over the forehead. A foam pad is put on the belly and fastened with straps. The $\mathrm{x}$-ray protection is used so that it does not interfere with the procedure. The only exceptions are enemas where $\mathrm{x}$-ray protection cannot be used, because it could cover or obscure the examined part of the bowel (1). Adjusted fluoroscopy technique should be kept in mind as described above (3).

\section{Fastening straps and sandbags}

The straps are used for younger children, or restless and excitable older children. They are made of non-radiopaque material with a stretching mechanism, which allows regulation of the strap pressure (1). The straps are fixed on both sides of the examination table and the child is fixed over the stomach or thighs. The child is protected with a lead rubber apron, placed so that it does not cover the part being examined. Different sizes of sandbags help to support and achieve symmetry of the examined part, and to prevent movement of the arms and legs when dealing with non-cooperative patients (1).

\section{Radiation protection equipment}

The concept of optimization refers to ensuring the quality of the equipment and using it in the optimal way, as well as minimizing radiation exposure, while maximizing image quality. Therefore the choice of radiographic procedure and how to perform it is crucial. The doses obtained at radiographic procedures differ depending on the type of the procedure and the way it is performed (3). The best results and least stress are obtained by introduction of some preventive measures. First of all, assuring a safe environment for children and other subjects involved in the procedure should be achieved by using the available $x$-ray protection, preventing exposure of pregnant girls (because of early sexual activity in teenagers - be alert with girls 13 or 14 years old) and mothers who bring their child for the examination. Good communication with a child and his/her parents is the next step to perform quality diagnostic imaging. It is necessary to obtain signed parental informed consent. The general principle for protection from radiation, known as ALARA (As Low As Reasonably Achievable), should be respected $(1,3)$. The exposure for radiation in $\mathrm{x}$-ray procedures must be as low as is reasonably achievable with consideration of the expected goals of the procedure, economy, and social factors.

Personal radiation protection equipment is made of lead and rubber, and is used to cover parts of body that are not in the primary beam, for the patient and the accompanying persons (1). It includes coats, aprons, gonad shields of various sizes, blankets, collars for thyroid protection, gloves, and glasses (1). Lead protection equipment should always be used during radiographic procedures: the choice of protection depends on the part being examined. It is important not to cover/ obscure the examined part, but all others parts must be protected. Lead protection must be at least $0.25 \mathrm{~cm}$ thick for staff and accompanying persons, and there must be at least $0.5 \mathrm{~cm}$ of lead shielding for any body part that may enter into the direct radiation beam which is not under investigation. For gonads, $1 \mathrm{~cm}$ of lead is used. 


\section{Conclusion}

Radiographers need to have various skills when working with pediatric patients. Each patient age group has its own complexity; therefore radiographers need expertise in more than just imaging procedures. Radiographers must always provide the necessary information and guidance to parents first to help keep the child still, particularly when the child is not cooperative. Patient safety is always in first place. The radiographic procedure (imaging techniques and exposure), radiation protection and immobilization equipment (prevention of unwanted movement) should be adjusted to the child's clinical condition and specific diagnostic issue. The result of such work is a lower radiation dose and higher quality radiograms, which allow clinicians to reach a diagnosis and prescribe the proper treatment.

Acknowledgment: We would like to thank assist. prof. Damjana Ključevšek, for her assistance and valuable suggestions during the planning and development of this article. Her help has been very much appreciated.

Authors' contributions: Conception and design: DK, DP; Acquisition, analysis and interpretation of data: DK, DP, NK; Drafting the article DP, DK; Revising it critically for important intellectual content: DK.

Conflict of interest: The authors declare that they have no conflict of interest.

\section{References}

1. Pašagić D. The radiological procedures at University children's hospital [undergraduate thesis]. Ljubljana: University of Ljubljana, Health faculty; 2004 .

2. Little MP. Risks associated with ionizing radiation. Br Med Bull. 2003;68(1):259-75.

3. Goske MJ, Charkot E, Herrmann T, John SD, Mills TT, Morrison G, et al. Image Gently: challenges for radiologic technologist when performing digital radiography in children. Pediatr Radiol. 2011;41(5):611-9.

4. Huda W. Radiation Doses and Risks in Chest Computed Tomography Examinations. Proc Am Thorac Soc. 2007;4(4):316-20.

5. Peck DJ, Samei E. How to Understand and Communicate Radiation Risk. ACR. 2010 Nov [updated 2014 July 22; cited 2015 Oct 27]; 1-23. Available from: http://www.imagewisely.org/imaging-modalities/computed-tomography/medicalphysicists/articles/how-to-understand-and-communicate-radiation-risk

6. Pediatric Chest X-rays. A Comparative Study of Dose Rates on Positioning in Pediatric chest $\mathrm{x}$ rays. Idaho: Idaho State University. c2011 - [updated 2011 Nov 15; cited 2015 Dec 2] 2015; 1-12. http://www.isu.edu/radsci/papers/100.pdf

7. Ward WL. Patient dose reduction during voiding cystourethrography. Pediatr Radiol. 2006; $9(2): 168-72$. 